\title{
Wireless power and data transfer through carbon composite using a common inductive link
}

\author{
Tuan Anh Vu ${ }^{1}$, Chi Van Pham ${ }^{2}$, William Tran ${ }^{3}$, Anh-Vu Pham ${ }^{4}$, Christopher S. Gardner ${ }^{5}$ \\ ${ }^{1,2,3,4}$ Department of Electrical and Computer Engineering, University of California, United State \\ ${ }^{5}$ Lawrence Livermore National Laboratory, United State \\ ${ }^{1}$ VNU University of Engineering and Technology, Vietnam
}

\section{Article Info}

\section{Article history:}

Received Apr 21, 2019

Revised Mar 21, 2020

Accepted May 6, 2020

\section{Keywords:}

Energy harvesting

Inductive coupling

Through carbon composite

Wireless data transfer

Wireless power transfer

\section{Corresponding Author:}

Tuan Anh Vu,

VNU University of Engineering and Technology,

144 Xuan Thuy Rd., Cau Giay Dist., Hanoi, Vietnam.

Email: tanhvu@vnu.edu.vn

\begin{abstract}
This paper presents the design and development of an integrated wireless power transfer and data communication system. The power and data transfer share a common inductive link that consists of two identical Helical coils placed on both sides of a carbon composite barrier. Power and data are transferred simultaneously through a 5-mm thick carbon composite barrier without any physical penetration or contact. Power transfer measurements show that the system can deliver $9.7 \mathrm{AC}$ power to the receiving coil with a power transfer efficiency of $36 \%$ through the carbon composite barrier. The system achieves a bidirectional half-duplex data communication with the data rate of unit $1.2 \mathrm{kbit} / \mathrm{s}$.
\end{abstract}

This is an open access article under the CC BY-SA license.

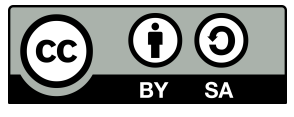

\section{INTRODUCTION}

Design and optimization of wireless power transfer (WPT) systems have been well studied over the last decades to charge cell phones and electric vehicles as well as to power up sensors over a short distance [1-6]. In recent years, there has been a great demand for wirelessly powered sensors to support structural health monitoring (SHM) for industrial applications containing carbon composite barriers and enclosures (e.g., naval vessel, aircraft and chemical vat). Drilling holes for feeding wires reduces the integrity of the structure. Specifically, practical issues include higher probabilities for the leakage of toxic chemicals and the loss of pressure or vacuum. The SHM systems typically require embedded sensors for data acquisition inside a sealed container, wireless communication, and energy harvesting. The sensors need to be powered and controlled wirelessly through a barrier without any physical penetration through mechanical structures.

Several methods have been proposed to transfer power and data wirelessly using a common inductive link. The concept of inductive power transfer (IPT) is similar to the principle of transformers in which an alternating magnetic field in the primary coil induces a load voltage on the secondary coil when the two coils are tightly coupled [7-9]. In low-power applications, the inductive link for power transfer is also used for data transmission, where the data is directly modulated on the power carrier [10-14]. However, these techniques have low data rates and low power transfers.

Several methods using multiple inductive links with multiple carriers have been proposed to increase the data rate while maintaining the power transfer efficiency $[15,16]$. In these methods, power and data are transferred via independent physical channels, where the power signal is delivered through one inductive 
link and the data signal is transmitted through another. However, multiple links will cause extra magnetic interferences between the two channels, thereby decreasing the signal-to-noise ratio (SNR) in a communication channel. Multiple inductive links also lead to larger device size, which is undesirable for confined space applications. The method with multiple carriers in a single inductive link is another candidate for WPT systems. Conventional systems feed both power and data signals through two terminals of the inductive coil [17, 18] or use strong couple inductors to mix them before driving those signals to the coil [19]. However, these approaches cause direct interference of high power leakage into the data communication band at multiple harmonics. As a result, sharp-response filtering circuits or high power data transmission (well above harmonics levels) is required for a successful data communication. The main challenge of this approach is to achieve a reliable communication link in the presence of a strong interference from the power link.

In this paper, a novel system for simultaneous power and data transfer through a 5-mm thick carbon composite barrier using a common inductive link will be presented [20, 21]. The block diagram of the proposed system is shown in Figure 1. In this system, power is applied two terminals of the transmitting coil while the data is fed through the coil's center tap. By doing this, the power and data signals are isolated by means of the half-coil impedance. This feeding configuration can help to reduce the data signal power being transmitted to only 1.5 . Consequently, it significantly releases the amount of power that needs to be harvested on the sensing side for sensing and communication operation. A complete system prototype is built to demonstrate that it can deliver 9.7 AC power to the sensing side and achieve wireless communication at the data rate of 1.2.

\section{SYSTEM OVERVIEW}

As demonstrated in Figure 1, the system is divided into two parts: the primary side and the sensing side. On the primary side, a power amplifier (PA) delivers power to two terminals of the transmitting coil while the data is applied via the coil's center tap. The power and data mixed signals are captured across two terminals of the receiving coils. A rectifier is employed on the sensing side to convert the harvested energy into DC power. This DC power is then used for sensing and communication operation on the sensing side, which does not have any batteries. The frequency of the power carrier is set at 300 while the data carrier is set at 8 . The data-carrier frequency should be at least an order of magnitude higher than the power-carrier frequency so that the crosstalk interference of data communication from power transfer is suppressed. This facilitates the design of demodulation circuit and guarantees the reliability of communication. Low frequency was chosen for the wireless power transfer to isolate high power leakage at multiple harmonics into the data communication band. Conversely, for the wireless data transfer, high frequency had to be chosen to have large bandwidth for high data rate. However, the frequency of data carrier cannot be too high because data signals may experience significant loss through the inductive link.

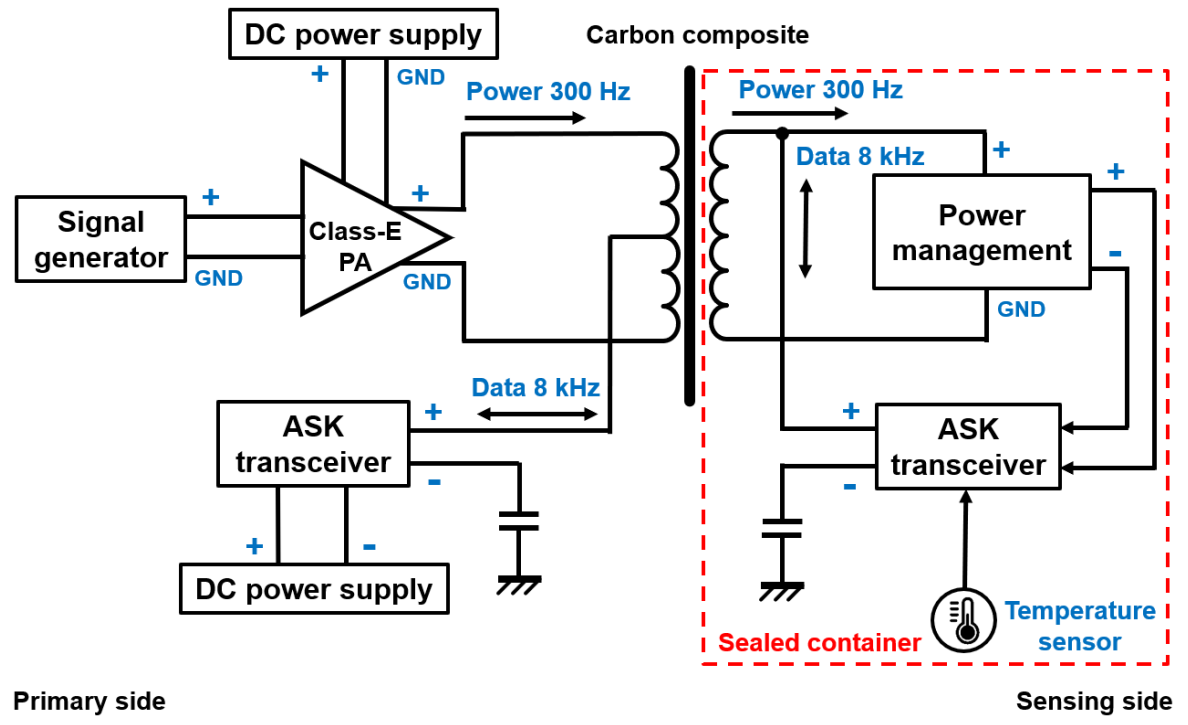

Figure 1. Proposed system for power and data transfer through carbon composite 


\subsection{ASK transceiver}

In the proposed system, bidirectional half-duplex communication between the primary side and the sensing side is implemented using amplitude-shift keying (ASK) modulation. The proposed ASK transceiver shown in Figure 2, is adopted in both sides. In forward communication, the primary side is set to a transmitter mode while the sensing side is set to a receiver mode. In backward communication, the primary side is set to a receiver mode while the sensing side is set to a transmitter mode. The ASK transceiver can operate in two modes: transmitter mode and receiver mode, which are controlled by a bidirectional electronics switch TPS2080 from Texas Instruments. On the transmitter mode, digital data from an Arduino microcontroller (uC) is mixed with an 8-kHz carrier frequency generated by a local oscillator (LO) on a printed circuit board (PCB). The ASK modulated signal is then amplified by a commercial audio PA TDA7391 from STMicroelectronics and employed as a signal source to transmit data. On the receiver mode, the incoming data is demodulated and read by the same Arduino microcontroller. The ASK demodulator includes an active high-pass filter (HPF), a squarer, a low-pass filter (LPF) and a comparator. The active HPF consists of a second-order RC HPF followed by an op-amp amplifier. The strong power carrier signal and its harmonics that may interfere with the baseband data signal are suppressed by 40 by the active HPF. The filtered signals are then amplified and demodulated by the following sub-blocks in the receiver chain.

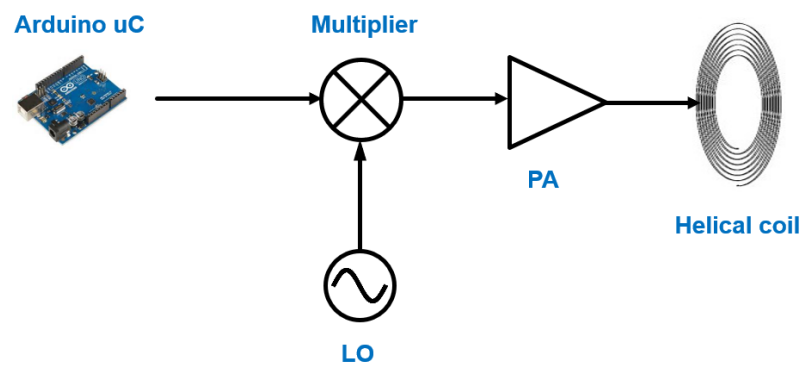

(a)

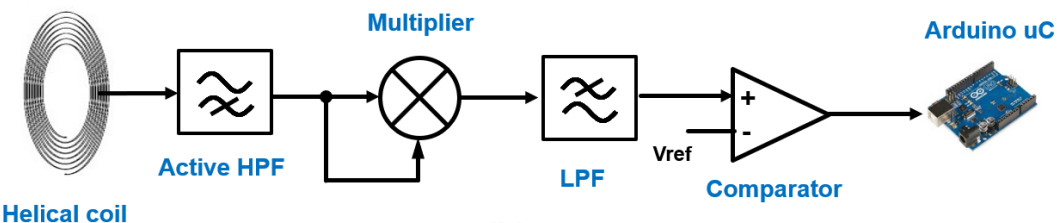

(b)

Figure 2. Proposed ASK transceiver, (a) ASK modulator, (b) ASK demodulator

\subsection{Class-E power amplifier}

A class-E PA is co-designed with the inductive link for power transfer. Class-E PAs achieve significantly higher efficiency than their conventional class-B or - $\mathrm{C}$ counterparts. The efficiency is maximized by minimizing power dissipation, while still maintaining a desired output power. The insulated-gate bipolar transistor (IGBT) STGW15S120DF3 from STMicroelectronics was used as the power transistor. This IGBT family has been specifically optimized for low switching frequencies. The complete circuit of the 300 class-E PA with all component values are given in Figure 3. The PA design is obtained by the Sokal-Raab approach [22, 23] is the coupling capacitor at the input of the PA. $R_{1}$ and $R_{2}$ form a voltage divider for gate biasing. The DC-feed choke, $L_{0}$ provides the connection to the DC power supply. $L_{1}$ and $L_{2}$ represent the coil inductances. In this design, the IGBT operates as an on/off switch and the load network shapes the voltage and current waveforms to prevent simultaneous high voltage and high current in the transistor. The load network is designed considering effects of the inductive link. The inductances of the transmitting and receiving coils become a part of the load network. $C_{1}$ and $C_{2}$ ensure that the collector voltage and collector current switching transitions are timedisplaced from each other. Consequently, the power dissipation is minimized, especially during the switching transitions. $L_{2}$ and $C_{3}$ form a series-parallel resonant circuit. The IGBT is driven with a gate voltage of 8 and a supply voltage of 15 . Measurement results show that the designed PA achieves a high collector efficiency of $54.2 \%$ when delivers 27 power at 300 to the transmitting coil. 


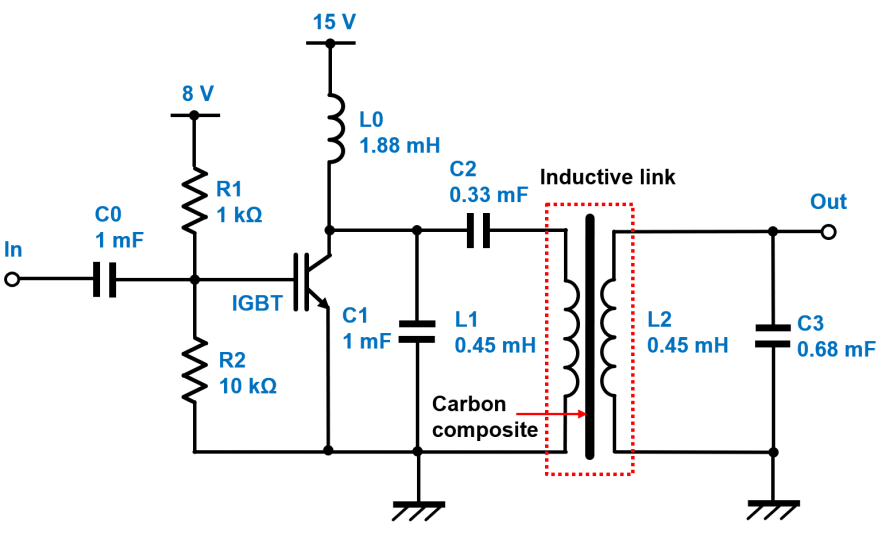

Figure 3. Schematic diagram of the class-E power amplifier

\subsection{Power management}

The power management module consists of an impedance transformer followed by several voltage regulators. Since the impedance of the coil is approximately 1 at 300 , the AC power is harvested on the receiving coil in form of low voltage and high current. The series-L parallel-C impedance transformer is used to boost up the harvested AC voltage. The AC voltage is then converted into the DC voltage by a full-bridge rectifier GBPC 3502W from ON Semiconductor followed by several smoothing capacitors. The DC voltage is distributed to several voltage regulators to generate stable voltage supplies for powering different sub-blocks of the ASK transceiver and sensors on the sensing side.

\subsection{Helical coil}

The power and data carrier share a common inductive link using a pair of identical Helical coils whose inductances are 0.45. Analysis and design of Helical coil were presented in [24-27] By co-axially aligning a pair of coils, the wireless IPT channel is formed without any physical penetration through the carbon composite barrier. The Helical circular geometry results in a more uniform magnetic field distribution that significantly improves the efficiency of energy transfer compared to that of the conventional Solenoid counterpart. The Helical coils are constructed using American Wire Gauge (AWG) 16 magnet wire. They have 63 turns with inner and outer radius of 22 and 108, respectively. Measurement results demonstrate that the power transfer efficiency is $36 \%$ for transfering power at 300 through the 5-mm thick carbon composite barrier.

\section{EXPERIMENTAL RESULTS}

A complete prototype was built to demonstrate the effectiveness of the proposed system. Figure 4 illustrates the measurement setup for power and data transfer through a $400 \times 400 \times 5$ carbon composite barrier. The other Helical coil cannot be seen in the figure since it is covered by the carbon composite barrier. For the practical demonstration of the proposed system, the prototype performances were verified in two experiments as shown in Figure 5. The first experiment verifies backward communication in which the sensing side sends data to the primary side. A temperature sensor TMP36 connected to the Arduino microcontroller on the sensing side continuously collects information on the temperature of the surrounding environment. The Arduino microcontroller is programmed to read this data and then the ASK transceiver sends the data to the primary side at a rate of 1.2. The temperature values are displayed on the monitor of a computer connected to the Arduino microcontroller board for further verification. In the second experiment, bidirectional half-duplex data communication is established when two ASK transceivers exchange one thousand bytes of data. The data is enclosed by the user-defined start and stop bytes. The ASK transceiver on the sensing side starts the communication at a data rate of 1.2 by sending the start byte followed by one thousand bytes of data and then the stop byte. After sending the stop byte, the transceiver is switched to receiver mode. On the primary side, as soon as the ASK transceiver detects the start byte, it is switched to the receiver mode and starts receiving the data in the backward communication. Alternatively, whenever the ASK transceiver on the primary side detects the stop byte, it is switched to the transmitter mode and starts sending the data in the forward communication. For both experiments, the ASK transceiver on the sensing side was powered by the rectified AC power that was harvested simultaneously while the data was received properly without any bit error. 


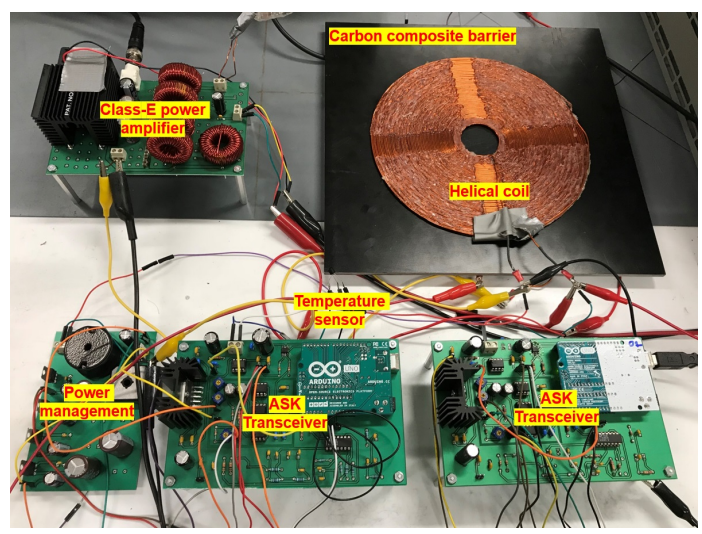

Figure 4. Measurement setup

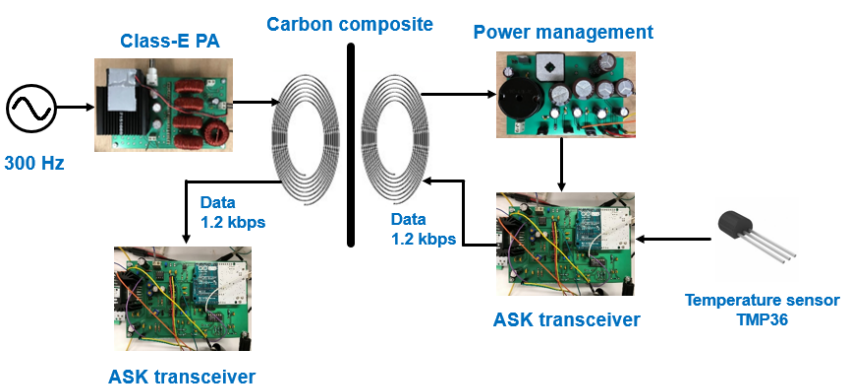

(a)

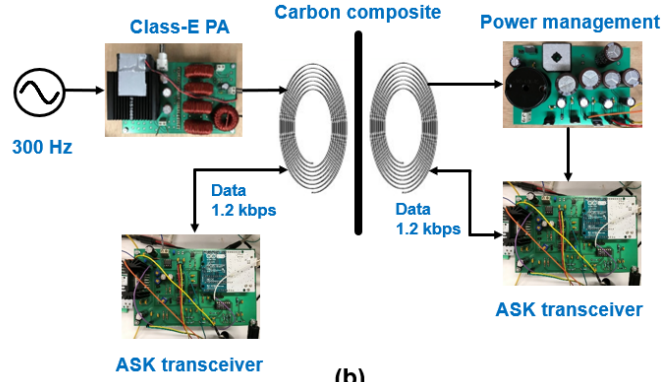

(b)

Figure 5. Experiment setups: (a) Real-time measurement of environment temperature; (b) Bidirectional half-duplex data communication.

Figure 6 (a) shows a typical spectrum of measured power and data mixed signals in the frequency domain captured by a vector signal analyzer. As seen in this figure, the modulated data signals exhibit lower side band (LSB) and upper side band (USB) symmetrically at the data-carrier frequency of 8 . At this data rate, the channel bandwidth needed for data communication is 2.4. The power of the data signal at the carrier frequency and LSB/USB are 14.6 and 6.1, respectively. At the fundamental frequency of 300, the power of transmitted signal is 30.3 while the second and third harmonics are 22.6 and 11.2, respectively. Although the power signal is much higher than the data signal, they are still separated when the data-carrier frequency is chosen to be much higher than the power-carrier frequency. Consequently, the modulated signal is not interfered with the high-order harmonics of the power-carrier signal. Figure 6 (b) shows the mixed signals in the time domain captured by an oscilloscope. The low frequency corresponds to the power-carrier signal of 300 , which is used to transfer power to the sensing side. The high frequency signal corresponds to the data-carrier signal of 8 , which is used for ASK modulation.

Figure 7 shows a measured signal waveforms in backward communication at a data rate of 1.2. The backward communication is more challenging due to the limited power budget that can be harvested on the sensing side. The demodulation of the weak reception data signals on the primary side is critical to have data received properly. Figure 7 (a) and 7 (b) show the transmitted digital data from the Arduino microcontroller and the ASK modulated signal after the data signal is mixed with the $8-\mathrm{kHz}$ carrier frequency generated by the local oscillator, respectively. The strong power-carrier signal at 300 and its harmonics are suppressed by the active HPF; thus, only data signals are available for demodulation. Figure 7c plots the baseband signals after downconversion and low-pass filtering. Depending on the received signal strength, the reference voltage of the following comparator is set accordingly. The digital data coming out from the ASK demodulator is plotted in Figure 7 (d). Comparing Figure 7 (a) with Figura 7 (d), it is demonstrated that the transmitted and received data are exactly the same with 0.24 delay time in transmission. The received digital data is read by the Arduino microcontroller on the primary side and displayed on a computer monitor for verification. The signal-to-noise 
ratio (SNR) of approximately 18 for a bandwidth of 2.4 could be obtained. Using the proposed system, sensors and communication circuits enclosed in sealed containers can be powered up and communicate with exterior devices without any physical penetration for wire feed-throughs.

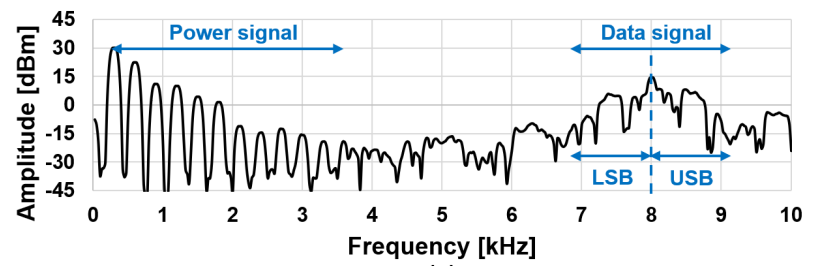

(a)

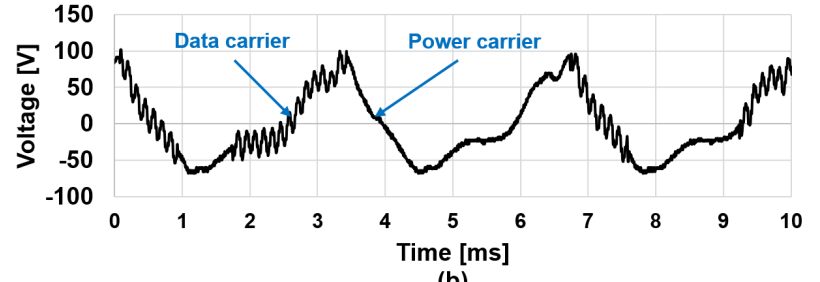

(b)

Figure 6. Measured transmitting mixed signals in, (a) frequency domain and (b) time domain
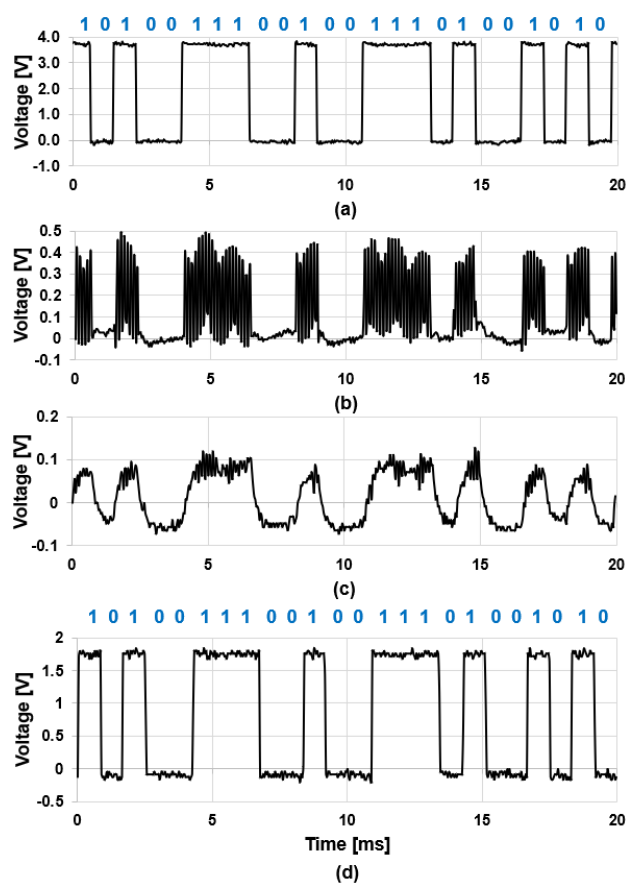

Figure 7. Measured signal waveforms in backward communication at data rate of 1.2, (a) Transmitted digital data, (b) ASK modulated signals before power amplification, (c) Received signal after low-pass filtering and

(d) Received digital data after thresholding

\section{CONCLUSION}

In this paper, we have presented a novel system using multiple carriers in a single inductive link for wireless power and data transfer through the 5-mm thick carbon composite barrier. The proposed system exploits the center tap architecture enabling high power transfer and data communication at the same time. Measurement results demonstrate that the system could achieve wireless communication at a data rate of 1.2 while the sensing side was powered up using 9.7 AC power that was harvested simultaneously. 


\section{ACKNOWLEDGMENT}

B620352.

This work has been supported by Lawrence Livermore National Laboratory under the project number

\section{REFERENCES}

[1] S. K. Oruganti and F. Bien, "Investigation of near-field wireless energy transfer for through metal-wall applications," 2014 IEEE Wireless Power Transfer Conference (WPTC), pp. 1-3, May 2014.

[2] Y.-S. Seo, Z. Hughes, M. Hoang, D. Isom, M. Nguyen, S. Rao and J.-C. Chiao,'Investigation of wireless power transfer in through-wall applications," 2012 Asia Pacific Microwave Conference Proceedings (APMC), pp. 1-4, December 2012.

[3] A. Peralta, S. H. Alizad, Nazmul Alam and Vinit Singh,'On the use of eddy currents to facilitate wireless power transfer through metallic surfaces," 2017 IEEE Wireless Power Transfer Conference (WPTC), pp. 1-3, May 2017.

[4] B. Minnaert and N. Stevens, "Maximizing the power transfer for a mixed inductive and capacitive wireless power transfer system," 2018 IEEE Wireless Power Transfer Conference (WPTC), pp. 1-4, June 2018.

[5] L. Huang, A. P. Hu, A. Swain, S. Kim and Y. Ren, "An overview of capacitively coupled power transfer-A new contactless power transfer solution," 8th IEEE Conference on Industrial Electronics and Applications (ICIEA), pp. 461-465, June 2013.

[6] C. Liu, A. P. Hu, B. Wang and N. C. Nair, "A capacitively coupled contactless matrix charging platform with soft switched transformer control," IEEE Transaction on Industrial Electronics, vol. 60, no. 1, pp. 249-260, January 2013.

[7] M. Yamakawa, Y. Mizuno, J. Ishida, K. Komurasaki and H. Koizumi, "Wireless power transmission into a space enclosed by metal walls using magnetic resonance coupling," Journal of Wireless Engineering and Technology, vol. 5, no. 1, pp. 19-24, January 2014.

[8] X. Li, C. Tang, X. Dai, P. Deng and Y. Su, "An inductive and capacitive combined parallel transmission of power and data for wireless power transfer systems," IEEE Transactions on Power Electronics, vol. 33, no. 6, pp. 4980-4991, June 2018.

[9] F. Lu, H. Zhang, H. Hofmann and C. C. Mi, "An inductive and capacitive integrated coupler and its LCL compensation circuit design for wireless power transfer," IEEE Transactions on Industry Applications, vol. 53, no.5, pp. 4903-4913, September/October, 2017.

[10] D.-X. Yang, Z. Hu, H. Zhao, H.-F. Hu, Y.-Z. Sun and B.-J. Hou, ”Through-metal-wall power delivery and data transmission for enclosed sensors: A review," MDPI Sensors Journal, vol. 15, pp. 31581-31605, December 2015.

[11] Y. Hu, X. Zhang, J. Yang and Q. Jiang, "Transmitting electric energy through a metal wall by acoustic waves using piezoelectric transducers," IEEE Transactions on Ultrasonics, Ferroelectrics, and Frequency Control, vol. 50, no. 7, pp. 773-781, July 2003.

[12] X. Bao, W. Biederman, S. Sherrit, M. Badescu, Y. Bar-Cohen, C. Jones, J. Aldrich and Z. Chang, "High-power piezoelectric acoustic-electric power feedthru for metal walls," The International Society for Optical Engineering (SPIE), vol. 6930, March 2008.

[13] D. J. Graham, J. A. Neasham and B. S. Sharif, "Investigation of methods for data communication and power delivery through metals," IEEE Transactions on Industrial Electronics, vol. 58, no. 10, pp. 4972-4980, October 2011.

[14] H. Zangl, A. Fuchs, T. Bretterklieber, M. J. Moser and G. Hollerl, "Wireless communication and power supply strategy for sensor applications within closed metal walls," IEEE Transaction on Instrumentation and Measurement, vol. 59, no. 6, pp. 1686-1692, June 2010.

[15] G. Simard, M. Sawan and D. Massicotte, "High-speed OQPSK and efficient power transfer through inductive link for biomedical implants," IEEE Transactions on Biomedical Circuits and System, vol. 4, no. 3, pp. 192-200, June 2010.

[16] T. Bieler, M. Perrottet, V. Nguyen, and Y. Perriard, "Contactless power and information transmission," IEEE Transactions on Industry Applications, vol. 38, no. 5, pp. 1266-1272, September/October, 2002.

[17] T. Drager, I. Mayordomo and J. Schuster, "Multi-band simultaneous inductive wireless power and data transmission," IEEE Sensors, pp. 1515-1518, November 2014.

[18] J. Hirai, T.-W. Kim and A. Kawamura, "Study on intelligent battery charging using inductive 
transmission of power and information," IEEE Transaction on Industrial Electronics, vol. 15, no. 2, pp. 335-345, March 2000.

[19] J. Wu, C. Zhao, Z. Lin, J. Du, Y. Hu and X. He, "Wireless power and data transfer via a common inductive link using frequency division multiplexing," IEEE Transaction on Industrial Electronics, vol. 62, no. 12, pp. 7810-7820, December 2015.

[20] T. A. Vu, C. V. Pham, A.-V. Pham and C. S. Gardner, "Wireless power transfer through metal using inductive link," International Journal of Power Electronics and Drive Systems (IJPEDS), vol. 10, no. 4, pp. 1-8, December 2019.

[21] C. V. Pham, T. A. Vu, W. Tran, A.-V. Pham and C. S. Gardner, "Wireless energy harvesting system through metal for aerospace sensor," 2018 IEEE Transportation Electrification Conference and Expo (ITEC), pp. 545-549, June 2018.

[22] N. O. Sokal, "Class-E switching-mode high-efficiency tuned RF/microwave power amplifier: Improved design equations," IEEE International Microwave Symposium (IMS), pp. 779-782, June 2000.

[23] F. H. Raab and N. O. Sokal, "Transistor power losses in the class E tuned power amplifier," IEEE Journal of Solid-State Circuits, vol. 13, no. 6, pp. 912-914, December 1978.

[24] C. V. Pham, A.-V. Pham and C. S. Gardner, "Development of helical circular coils for wireless through-metal inductive power transfer," IEEE Wireless Power Transfer Conference (WPTC), pp. 1-3, May 2017.

[25] M. Kiani and M. Ghovanloo, "The circuit theory behind coupled-mode magnetic resonance-based wireless power transmission," IEEE Transaction on Circuits and Systems I: Regular papers, vol. 59, no. 9, pp. 2065-2074, September 2012.

[26] C. J. Chen, T. H. Chu, C. L. Lin, and Z. C. Jou, ”A study of loosely coupled coils for wireless power transfer," IEEE Transaction on Circuits and Systems II: Express Briefs, vol. 57, no. 7, pp. 536-540, July 2010.

[27] C. R. Sullivan, "Computationally efficient winding loss calculation with multiple windings, arbitrary waveforms, and two-dimensional or threedimensional field geometry," IEEE Transactions on Power Electronics, vol. 16, no. 1, pp. 142-150, January 2001. 